\title{
Identification of distinct genes associated with seawater aspiration-induced acute lung injury by gene expression profile analysis
}

\author{
WEI LIU, LEI PAN, MINLONG ZHANG, LIYAN BO, CONGCONG LI, \\ QINGQING LIU, LI WANG and FAGUANG JIN
}

Department of Pulmonary Diseases, Tangdu Hospital, Fourth Military Medical University, Xi'an, Shaanxi 710038, P.R. China

Received July 7, 2015; Accepted July 7, 2016

DOI: $10.3892 / \mathrm{mmr} .2016 .5607$

\begin{abstract}
Seawater aspiration-induced acute lung injury (ALI) is a syndrome associated with a high mortality rate, which is characterized by severe hypoxemia, pulmonary edema and inflammation. The present study is the first, to the best of our knowledge, to analyze gene expression profiles from a rat model of seawater aspiration-induced ALI. Adult male Sprague-Dawley rats were instilled with seawater $(4 \mathrm{ml} / \mathrm{kg})$ in the seawater aspiration-induced ALI group (S group) or with distilled water $(4 \mathrm{ml} / \mathrm{kg})$ in the distilled water negative control group (D group). In the blank control group (C group) the rats' tracheae were exposed without instillation. Subsequently, lung samples were examined by histopathology; total protein concentration was detected in bronchoalveolar lavage fluid (BALF); lung wet/dry weight ratios were determined; and transcript expression was detected by gene sequencing analysis. The results demonstrated that histopathological alterations, pulmonary edema and total protein concentrations in BALF were increased in the $\mathrm{S}$ group compared with in the D group. Analysis of differential gene expression identified up and downregulated genes in the $\mathrm{S}$ group compared with in the D and C groups. A gene ontology analysis of the differential gene expression revealed enrichment of genes in the functional pathways associated with neutrophil chemotaxis, immune and defense responses, and cytokine activity. Kyoto Encyclopedia of Genes and Genomes analysis revealed that the cytokine-cytokine receptor interaction pathway was one of the most important pathways involved in seawater aspiration-induced ALI. In conclusion, activation of the cytokine-cytokine receptor interaction pathway may have an essential role in the progression of seawater
\end{abstract}

Correspondence to: Professor Faguang Jin, Department of Pulmonary Diseases, Tangdu Hospital, Fourth Military Medical University, 569 Xinsi Road, Xi'an, Shaanxi 710038, P.R. China E-mail: jinfag@fmmu.edu.cn

Key words: seawater aspiration-induced ALI, gene expression profiles, TNFSF10, IL-6 aspiration-induced ALI, and the downregulation of tumor necrosis factor superfamily member 10 may enhance inflammation. Furthermore, IL-6 may be considered a biomarker in seawater aspiration-induced ALI.

\section{Introduction}

Drowning is one of the most common causes of accidental mortality worldwide, and remains a significant medical and social issue (1). Secondary acute lung injury (ALI) is a serious complication of drowning. Seawater aspiration-induced ALI, which has a rapid progress and high mortality rate, is a common complication of drowning in seawater (2). Seawater aspiration-induced ALI exhibits similar characteristics to stress situations, including trauma, burns and sepsis-induced $\mathrm{ALI}$, and is characterized by an acute inflammatory response in the pulmonary parenchyma and interstitial tissue, severe hypoxemia and pulmonary edema (3). Previous studies indicated that inflammatory cell infiltration (4-7) and permeability of the alveolar wall (8) were increased in a rat model of seawater aspiration-induced ALI, and apoptosis of alveolar epithelial cells was also involved in seawater aspiration-induced ALI (9). Another of our previous studies investigated the role of estrogen in a rat model of seawater aspiration-induced ALI. The results demonstrated that treatment with estrogen was able to protect against early stage seawater aspiration-induced ALI (10). It is well-known that nuclear factor (NF)- $\kappa \mathrm{B}$ is an important molecule associated with sepsis-induced ALI (11). Recently, it has been reported that NF-kB and RhoA/Rho kinase pathways are critical in the progression of seawater aspiration-induced ALI (12). However, whether the inflammatory response is important in the pathogenesis and potential molecular mechanisms of seawater aspiration-induced ALI remains to be elucidated.

To elucidate the potential molecular mechanisms underlying seawater aspiration-induced ALI, a gene expression profile analysis was conducted on lungs obtained from a rat model using Illumina Solexa sequencing technology. A large number of genes were compared between the experimental groups. In order to explore the potential molecular mechanisms underlying seawater aspiration-induced ALI, a distilled water group was used as a negative control, and a blank control 
Table I. Assigned lung injury scores in the seawater aspiration-induced acute lung injury group and the control groups.

\begin{tabular}{llllcr}
\hline Group & Edema & $\begin{array}{c}\text { Neutrophil } \\
\text { infiltration }\end{array}$ & Hemorrhage & $\begin{array}{c}\text { Bronchiole epithelial } \\
\text { desquamation }\end{array}$ & $\begin{array}{c}\text { Hyaline } \\
\text { membrane }\end{array}$ \\
\hline Blank control & $0.1 \pm 0.16$ & $0.2 \pm 0.26$ & $0.1 \pm 0.15$ & $0.2 \pm 0.23$ & $0.0 \pm 0.00$ \\
Distilled water negative control & $3.0 \pm 0.15^{\mathrm{a}, \mathrm{b}}$ & $3.2 \pm 0.17^{\mathrm{a}, \mathrm{b}}$ & $2.2 \pm 0.25^{\mathrm{a}, \mathrm{b}}$ & $2.7 \pm 0.28^{\mathrm{a}, \mathrm{b}}$ & $0.2 \pm 0.24$ \\
Seawater & $3.7 \pm 0.24^{\mathrm{a}}$ & $3.6 \pm 0.25^{\mathrm{a}}$ & $3.1 \pm 0.37^{\mathrm{a}}$ & $3.5 \pm 0.36^{\mathrm{a}}$ & $0.3 \pm 0.36$ \\
\hline
\end{tabular}

Data are presented as the mean \pm standard error of the mean. ${ }^{\mathrm{a}} \mathrm{P}<0.05$ vs. the blank control group; ${ }^{\mathrm{b}} \mathrm{P}<0.05$ vs. the seawater group; $\mathrm{n}=3$.

A

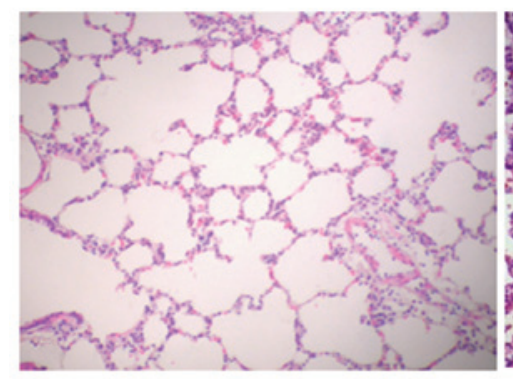

B

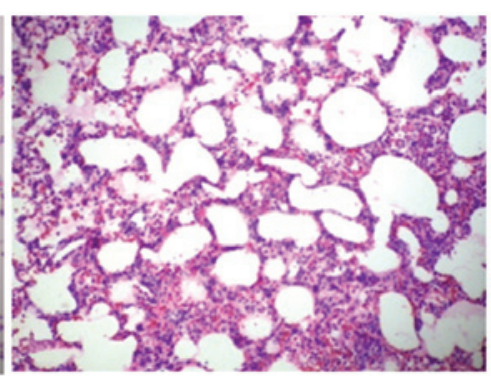

$\mathbf{C}$

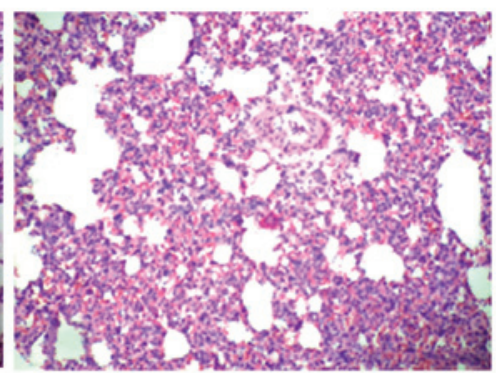

Figure 1. Histopathological alterations in seawater aspiration-induced acute lung injury (ALI) (hematoxylin-eosin staining; original magnification, 200x). (A) Blank control group: Lung structure was normal; (B) distilled water negative control group: Alveolar wall was thicker, edema and hemorrhage were detected, alveolar space was reduced, and obvious inflammatory cell infiltration was detected in the interstitial and alveolar space; (C) seawater aspiration-induced ALI group, alterations were similar to the distilled water negative control group but were more severe.

$\mathbf{A}$

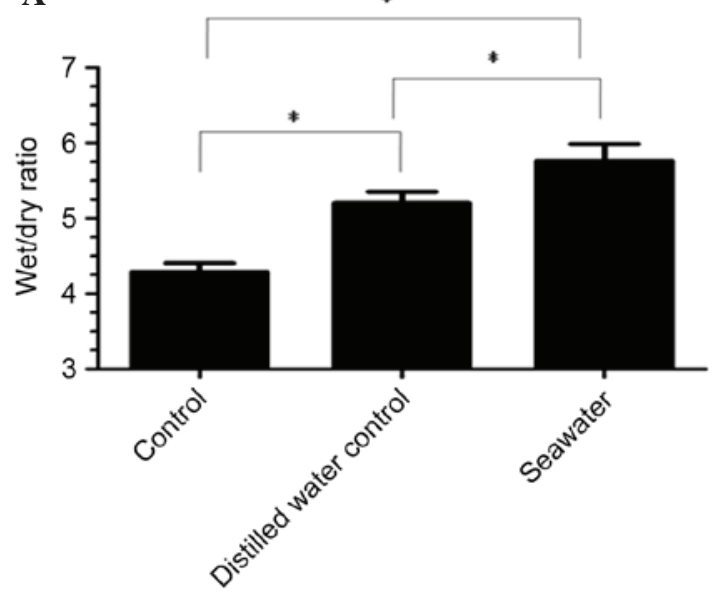

B

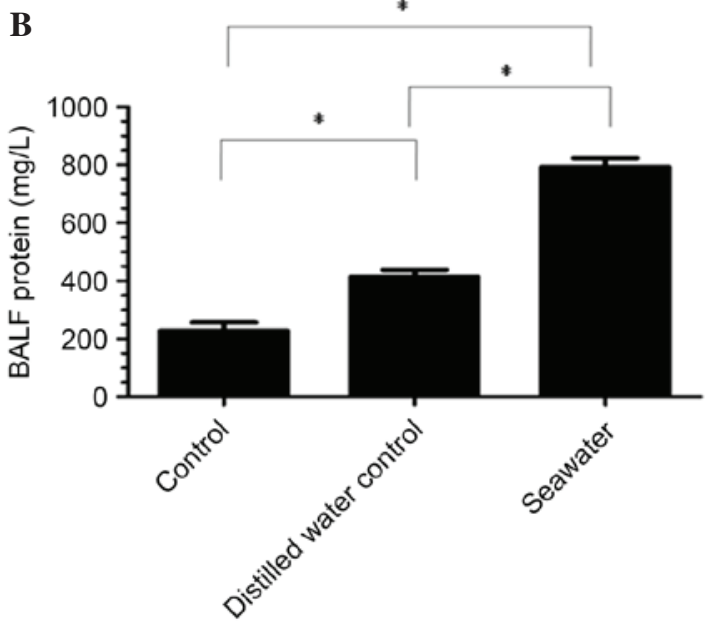

Figure 2. (A) Lung wet/dry ratios and (B) total protein concentrations in bronchoalveolar lavage fluid (BALF) were altered in the seawater aspiration-induced acute lung injury group. Data are presented as the mean \pm standard error of the mean $(\mathrm{n}=3)$. ${ }^{*} \mathrm{P}<0.05$.

group was also established. Gene expression profiles were compared in an effort to identify unique gene classifiers and to differentiate between the three groups. The present study is the first, to the best of our knowledge, to use gene expression profile analysis to explore the potential molecular mechanisms underlying seawater aspiration-induced ALI.

\section{Materials and methods}

Animals and materials. Adult male Sprague-Dawley rats (weight, 180-220 g; age, 6 weeks) were obtained from the
Animal Center of the Fourth Military Medical University (Xi'an, China). The rats were housed in filtered, temperature-controlled units $\left(24^{\circ} \mathrm{C}, 50 \%\right.$ humidity), with ad libitum access to food and water and with diurnal variation of light. The experiments were approved by the Animal Care and Use Committee of the Fourth Military Medical University, and were conducted in accordance with the Declaration of the National Institutes of Health Guide for Care and Use of Laboratory Animals (Publication No. 85-23; revised 1985). Seawater (osmolality, 1,300 mmol/1; $\mathrm{pH} 8.2$; specific gravity, 1.05; $\mathrm{NaCl}, 26.518 \mathrm{~g} / \mathrm{l} ; \mathrm{MgSO}_{4}, 3.305 \mathrm{~g} / \mathrm{l} ; \mathrm{MgCl}_{2}, 2.447 \mathrm{~g} / \mathrm{l}$; 
Table II. Top 10 up and downregulated genes in the seawater aspiration-induced acute lung injury group compared with the blank control group.

\begin{tabular}{clcccc}
\hline No. & \multicolumn{1}{c}{ Gene } & S (FPKM) & C (FPKM) & Fold change $^{\mathrm{a}}$ & P-value $^{2}$ \\
\hline Up & & & & & \\
1 & Cxc12 & 158.856 & 0.935096 & 169.89 & $<0.01$ \\
2 & F1M1Q7_RAT & 615.196 & 4.13873 & 148.63 & $<0.01$ \\
3 & Orm1 & 104.343 & 0.718 & 145.32 & $8.49 \mathrm{E}-12$ \\
4 & Irg1 & 75.7689 & 0.635845 & 119.16 & $<0.01$ \\
5 & B1H259_RAT & 5.58517 & 0.091918 & 60.76253 & $5.75 \mathrm{E}-05$ \\
6 & Cxc16 & 110.903 & 2.12643 & 52.15451 & $7.89 \mathrm{E}-08$ \\
7 & D3ZDX3_RAT & 19.8754 & 0.389918 & 50.9733 & 0.000122 \\
8 & Csf3 & 9.44276 & 0.186485 & 50.6356 & 0.000126 \\
9 & Fcrls & 117.251 & 2.67019 & 43.91129 & $<0.01$ \\
10 & Tnn & 6.79358 & 0.169375 & 40.10972 & $2.41 \mathrm{E}-06$ \\
Down & & & & \\
1 & F1LVW9_RAT & 0.561806 & 14.1462 & 25.17989 & 0.002012 \\
2 & Rnase17 & 1.16126 & 21.15 & 18.21292 & 0.038443 \\
3 & D3Z9Q1_RAT & 2.15125 & 37.4138 & 17.39161 & $1.06 \mathrm{E}-09$ \\
4 & Vipr1 & 12.7842 & 196.93 & 15.40418 & $1.21 \mathrm{E}-07$ \\
5 & Ear11 & 4.0495 & 61.6461 & 15.2232 & $4.98 \mathrm{E}-10$ \\
6 & Slc62 & 0.61605 & 7.91125 & 12.84193 & $3.46 \mathrm{E}-06$ \\
7 & Nova2 & 0.458669 & 5.88786 & 12.83686 & $5.59 \mathrm{E}-05$ \\
8 & Csrp3 & 0.649324 & 8.33526 & 12.83686 & 0.000771 \\
9 & F1M5M6_RAT & 8.74595 & 111.67 & 12.76826 & $2.96 \mathrm{E}-07$ \\
10 & Cyp1a1 & 2.47813 & 29.744 & 12.00256 & $1.07 \mathrm{E}-11$ \\
\hline
\end{tabular}

${ }^{\text {a}}$ Fold change values are calculated by dividing the $\mathrm{S}$ group values by the $\mathrm{C}$ group values. S, seawater group; C, blank control group; FPKM, fragments per kilobase of exon per million fragments mapped.

$\mathrm{CaCl}_{2}, 1.141 \mathrm{~g} / \mathrm{l} ; \mathrm{KCl}, 0.725 \mathrm{~g} / \mathrm{l} ; \mathrm{NaHCO}_{3}, 0.202 \mathrm{~g} / \mathrm{l} ; \mathrm{NaBr}$, $0.083 \mathrm{~g} / \mathrm{l}$ ) was prepared according to the major composition of the East China Sea provided by the Chinese Ocean Bureau (Beijing, China). Distilled water (osmolality, $0 \mathrm{mmol} / \mathrm{l}$ ) was prepared according to distilled water preparation.

Animal modeling and grouping. The seawater aspiration model was produced according to the method described previously (10), with some modifications. The rats were anesthetized with $3 \%$ pentobarbital sodium $(1.5 \mathrm{ml} / \mathrm{kg}$ of body weight; intraperitoneal administration) prior to exposure of the tracheae.

Sprague-Dawley rats were randomly assigned into three groups ( $\mathrm{n}=3$ /group): Blank control group (C group), distilled water negative control group (D group) and seawater aspiration-induced ALI group (S group). Seawater or distilled water $(4 \mathrm{ml} / \mathrm{kg}$ ) was instilled into the rat lungs via the trachea in $4 \mathrm{~min}$ at a steady speed using a $2 \mathrm{ml}$ syringe. In the blank control group the rats' tracheae were exposed without instillation.

RNA isolation and lung histopathology. The rats were sacrificed by aortic transection $6 \mathrm{~h}$ after tracheal exposure. Rats were anesthetized with $3 \%$ pentobarbital sodium $(1.5 \mathrm{ml} / \mathrm{kg}$ of body weight; intraperitoneal administration) prior to aortic transection. A section from the right lower lobe of the lung was removed from each rat, immediately frozen and stored in liquid nitrogen until further use. RNA extraction, and quantification and quality assessment of the extracted RNA was performed as previously described (13). Another section from the right lower lobe of the lung was fixed with $4 \%$ paraformaldehyde for $24 \mathrm{~h}$. The tissues were then embedded in paraffin and cut into $5 \mu \mathrm{m}$ sections. Hematoxylin-eosin staining was performed according to a standard protocol (14).

Histopathological quantification. Three samples from each group underwent quantification of lung histopathology. The sections were stained with hematoxylin-eosin and were analyzed independently by two pathologists who were blind to the groups the animals belonged to. After viewing $\sim 10$ fields per section at low and high magnification with a light microscope, each section was given a lung injury score, which ranged between 0 and 4 , based on edema, neutrophil infiltration, hemorrhage, bronchiole epithelial desquamation and hyaline membrane formation, according to a previously described standard assessment (15). The scores (0 to 4 ) represent severity of lung injury: 0 , no injury (normal appearing lung); 1, modest injury (limited congestion and edema, but no interstitial neutrophil infiltration with occasional red blood cells and neutrophils in the alveolar spaces); 2 , intermediate injury (mild congestion, edema, and interstitial neutrophil infiltration with occasional red blood cells and neutrophils 
Table III. Top 10 up and downregulated genes in the seawater aspiration-induced acute lung injury group compared with the distilled water negative control group.

\begin{tabular}{clcccc}
\hline No. & Gene & S (FPKM) & D (FPKM) & Fold change $^{\mathrm{a}}$ & P-value $^{2}$ \\
\hline Up & & & & & \\
1 & F1M1Q7_RAT & 615.196 & 2.90533 & 211.7473 & $<0.01$ \\
2 & F1M663_RAT & 358.937 & 9.77567 & 36.71735 & $8.44 \mathrm{E}-15$ \\
3 & Il6 & 26.1417 & 1.0887 & 24.01194 & $1.34 \mathrm{E}-09$ \\
4 & D4A7S8_RAT & 79.1362 & 3.4686 & 22.81499 & $1.69 \mathrm{E}-06$ \\
5 & Irg1 & 75.7689 & 3.44331 & 22.0047 & $<0.01$ \\
6 & F1LWN4_RAT & 112.483 & 5.1964 & 21.64617 & $9.04 \mathrm{E}-08$ \\
7 & Frrls & 117.251 & 5.88037 & 19.9394 & $<0.01$ \\
8 & Slamf9 & 183.298 & 10.7154 & 17.10599 & $4.44 \mathrm{E}-16$ \\
9 & LOC100363638 & 38.4476 & 2.44753 & 15.70868 & 0.000528 \\
10 & Atp6v0a4 & 5.02422 & 0.339452 & 14.80097 & $4.02 \mathrm{E}-06$ \\
Down & & & & & \\
1 & Cyp1a1 & 2.47813 & 51.4444 & 20.75936 & $2.22 \mathrm{E}-16$ \\
2 & F1LVW9_RAT & 0.561806 & 9.76356 & 17.37884 & 0.000146 \\
3 & F1MAE7_RAT & 25.1881 & 393.779 & 15.63362 & $2.43 \mathrm{E}-13$ \\
4 & Wnk2 & 0.279078 & 4.32902 & 15.51186 & $8.09 \mathrm{E}-08$ \\
5 & Vipr1 & 12.7842 & 164.067 & 12.83365 & $9.70 \mathrm{E}-07$ \\
6 & ENSRNOG00000030444 & 1.37746 & 15.7838 & 11.45861 & $3.90 \mathrm{E}-07$ \\
7 & Ear11 & 4.0495 & 43.4886 & 10.73924 & $8.88 \mathrm{E}-08$ \\
8 & Nr1d1 & 3.18831 & 32.3391 & 10.14298 & 0.000344 \\
9 & LOC100365881 & 1.44117 & 13.8846 & 9.634234 & $8.34 \mathrm{E}-06$ \\
10 & Zbtb16 & 6.00713 & 54.2278 & 9.027272 & $4.31 \mathrm{E}-11$ \\
\hline
\end{tabular}

${ }^{\text {a } F o l d ~ c h a n g e ~ v a l u e s ~ a r e ~ c a l c u l a t e d ~ b y ~ d i v i d i n g ~ t h e ~} \mathrm{~S}$ group values by the $\mathrm{D}$ group values. S, seawater group; D, distilled water negative control group; FPKM, fragments per kilobase of exon per million fragments mapped.

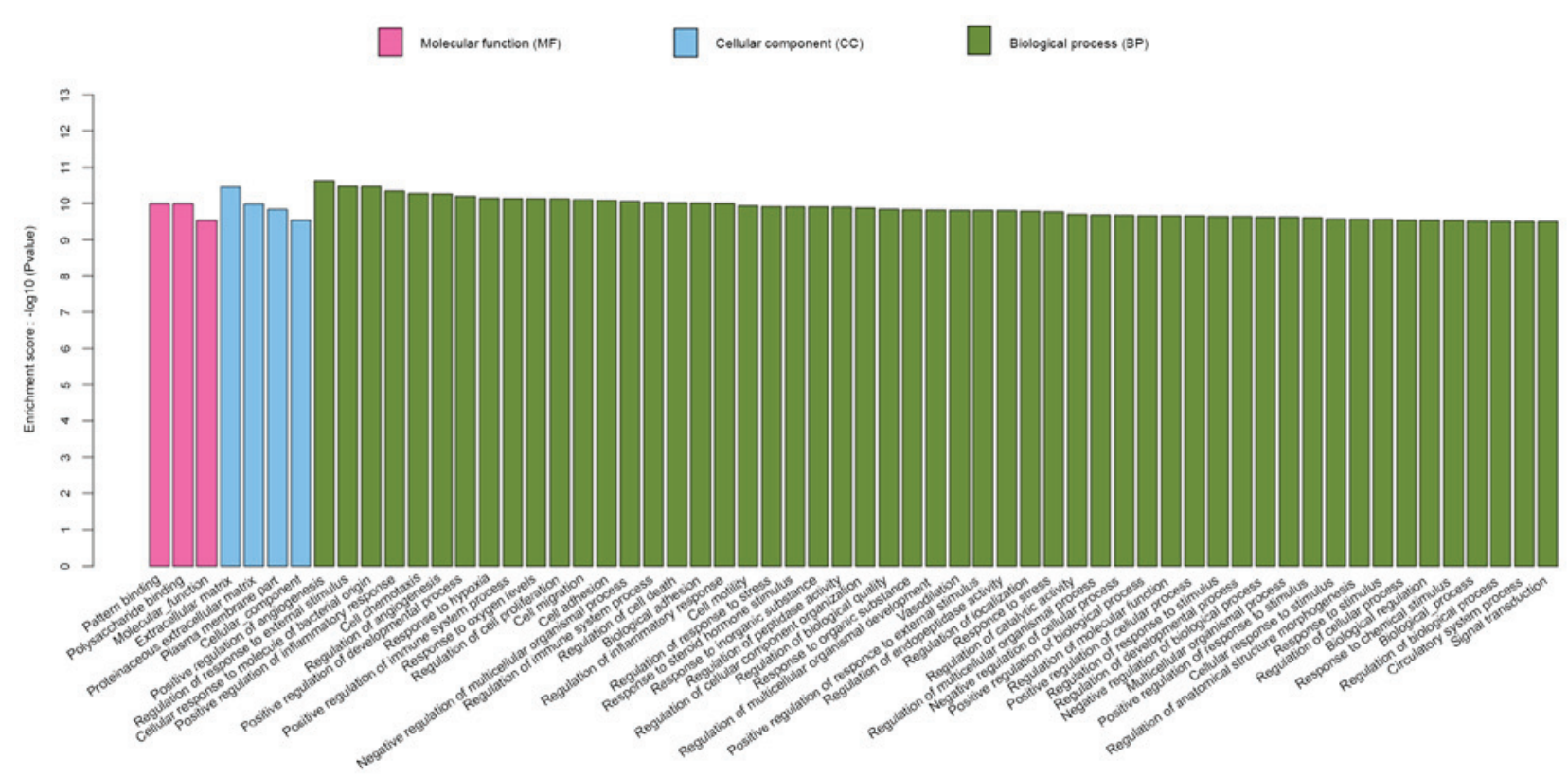

Figure 3. Enrichment of the total identified genes in the seawater (S) group according to gene ontology (GO) annotation, including molecular function, cellular component and biological process. These GO terms were not enriched in the distilled water (D) group.

in the alveolar spaces); 3 , widespread injury (more prominent congestion and edema, with neutrophils partially filling the alveolar spaces but with consolidation); and 4, widespread injury (most prominent, marked congestion and edema, with 
neutrophilic infiltrate nearly filling the alveolar spaces or with frank lung consolidation) (16).

Lung wet/dry ratios. To quantify the magnitude of pulmonary edema, the wet/dry weight ratios of the lung samples from the upper and middle lobes of the right lung from each rat were determined. Tissue samples were obtained $6 \mathrm{~h}$ after instillation, were immediately weighed, and were then subjected to desiccation in an oven at $70^{\circ} \mathrm{C}$ until a stable dry weight was achieved after $72 \mathrm{~h}$. The wet/dry weight ratios were calculated, in order to quantify the magnitude of pulmonary edema (14).

Preparation of bronchoalveolar lavage fluid (BALF). Rats were sacrificed $6 \mathrm{~h}$ after instillation, then the left lungs $(\mathrm{n}=3)$ were excised from the rats, and BALF was collected using intratracheal injections of $2 \mathrm{ml}$ ice-cold phosphate-buffered saline. More than $90 \%$ of the BALF was recycled. Subsequently, BALF was centrifuged $(12,000 \mathrm{x} \mathrm{g}, 10 \mathrm{~min}$, $4^{\circ} \mathrm{C}$ ), and protein concentrations in BALF were determined according to Lowry's method (17), with bovine serum albumin as a standard.

Sequencing analysis. One biological replicate was tested from each group. To minimize the impact of interindividual variability on the sequencing data, each biological replicate consisted of pooled lung RNA samples prepared from three rats per group: $\mathrm{C}$ group sample (a pooled sample from three blank control samples), D group sample (a pooled sample from three distilled water negative control samples), and $\mathrm{S}$ group sample (a pooled sample from three seawater aspiration-induced ALI samples).

The Illumina standard kit was used for mRNA-Seq sample preparation, according to the manufacturer's protocol (Illumina, San Diego, CA, USA). Sequencing was performed on a Genome Analyzer (GAII; Illumina) using a 50-bp read length. The sequence reads were processed and contrasted to the Ensembl (http://asia.ensembl.org/info/data/ftp/index.html) rat reference genome using TopHat (http://ccb.jhu.edu/software/tophat/index.shtml). The relative abundance of genes was measured using Cufflinks (http://cole-trapnell-lab.github. io/cufflinks/) compared with normalized RNA-Seq fragments. The unit of measurement is fragments per kilobase of exon per million fragments mapped (FPKM). The confidence intervals for the FPKM estimates were calculated according to a Bayesian inference method. The differentially expressed genes were visualized using the CummeRbund program (http://compbio.mit.edu/cummeRbund/).

Gene products that were $\geq 2$-fold differentially expressed were further analyzed, as described in the results section. Differentially expressed genes were classified according to their gene ontology (GO; http://www.geneontology.org/), using goatools (https://github.com/tanghaibao/goatools). Cellular pathway association was analyzed according to the Kyoto Encyclopedia of Genes and Genomes (KEGG) database (http://www.genome.jp/kegg/) using the KEGG Orthology Based Annotation System (http://kobas.cbi.pku.edu.cn/home. do). The sequencing data have been deposited in the National Center for Biotechnology Information short read archive (http://www.ncbi.nlm.nih.gov/Traces/sra/sra.cgi) under the accession number SRP052721. 


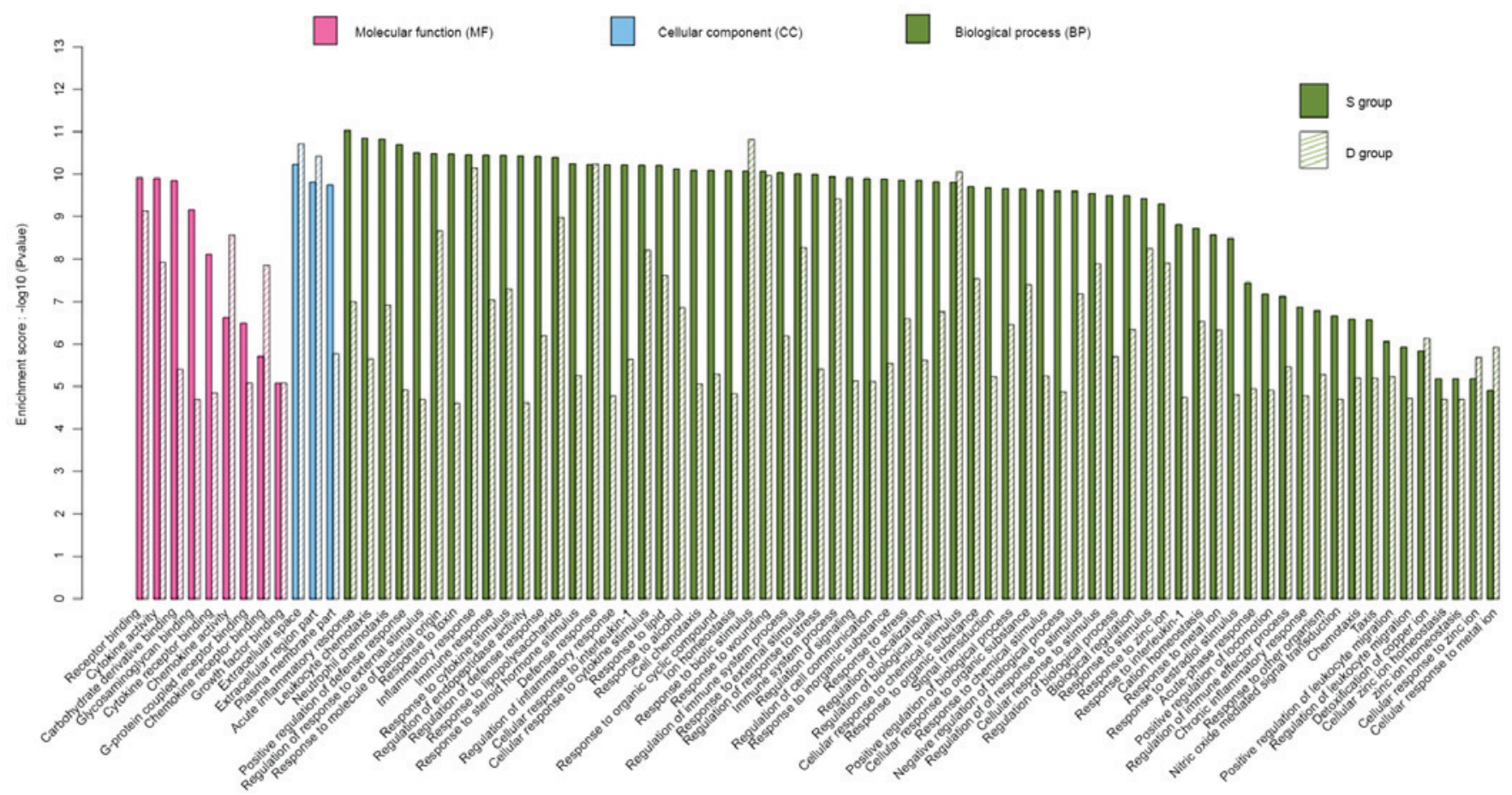

Figure 4. Gene ontology terms enriched in the seawater (S) and distilled water (D) groups.

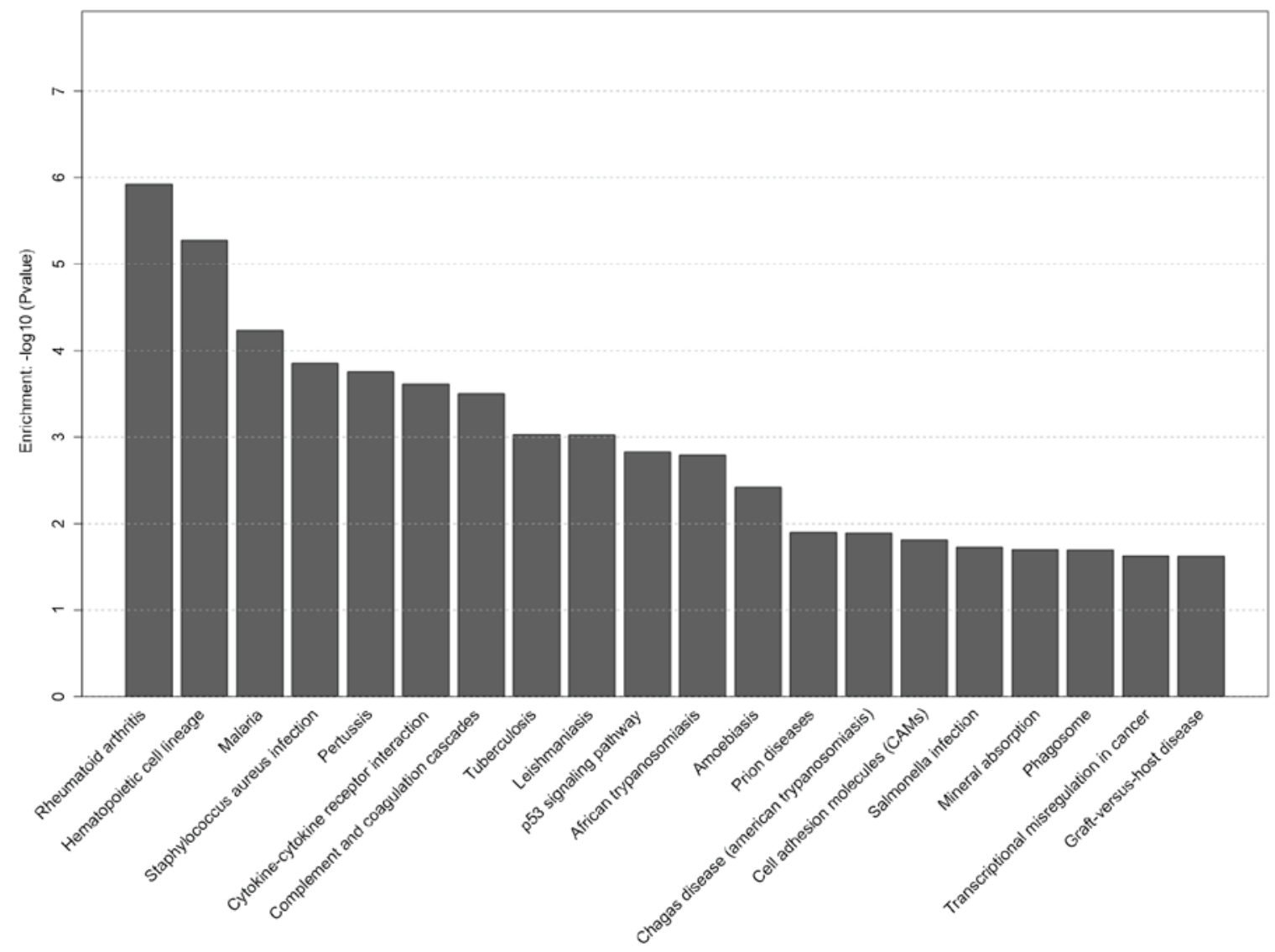

Figure 5. Top 20 pathways with significantly differentially expressed genes in the seawater (S) group.

Reverse transcription-quantitative polymerase chain reaction (RT-qPCR). RT was performed on total RNA (200 ng) in a total volume of $13 \mu \mathrm{l}$ containing $1 \mu \mathrm{l} 50 \mu \mathrm{M}$ random hexamers, $1 \mu 110 \mathrm{mM}$ dNTP mix, DEPC water. RNA-primer mix was incubated at $65^{\circ} \mathrm{C}$ for $5 \mathrm{~min}$, then incubated on ice for $>1$ min. $5 X$ SuperScript IV Buffer $(4 \mu 1$; Thermo Fisher 


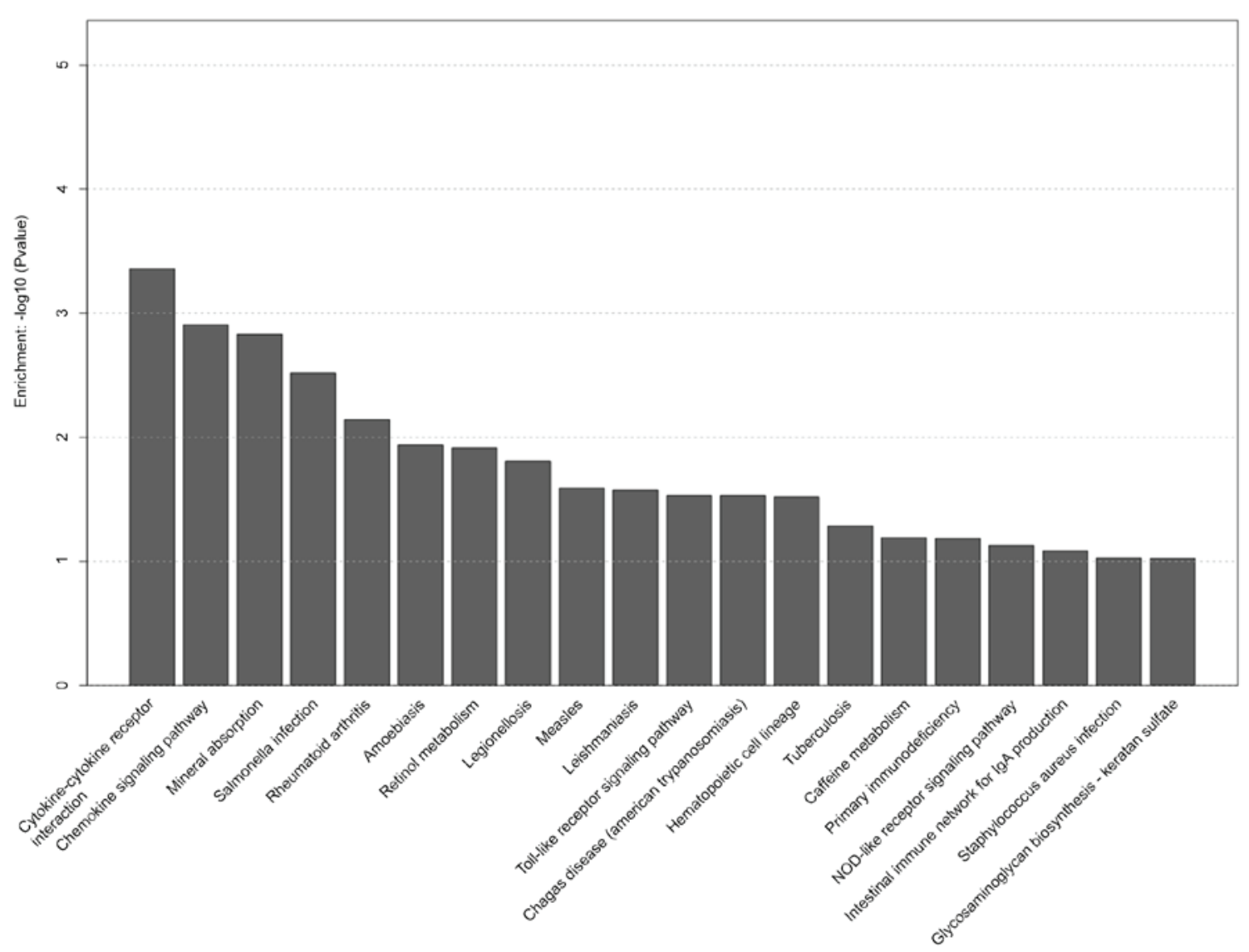

Figure 6. Top 20 pathways with significantly differentially expressed genes in the distilled water (D) group.

Scientific, Inc., Waltham, MA, USA), $100 \mathrm{mM}$ dithiothreitol $(1 \mu \mathrm{l})$, RNase inhibitor $(1 \mu \mathrm{l})$ and $200 \mathrm{U} / \mu 1$ SuperScript Reverse Transcriptase ( $1 \mu \mathrm{l}$; Thermo Fisher Scientific, Inc.) were added to the reaction. The samples were incubated at $23^{\circ} \mathrm{C}$ for $10 \mathrm{~min}, 50^{\circ} \mathrm{C}$ for $10 \mathrm{~min}$, and $80^{\circ} \mathrm{C}$ for $10 \mathrm{~min}$. qPCR reactions were performed using the QuantiTect SYBR Green PCR kit (Qiagen, Inc., Valencia, CA, USA) to detect the mRNA expression levels of the following genes: C-X-C motif chemokine ligand (CXCL)2, CXCL13, C-C motif chemokine ligand (CCL)20, CCL3, interleukin (IL)-6, IL-1 receptor type 2 (IL1R2) and tumor necrosis factor superfamily member 10 (TNFSF10). Each value obtained is the mean of three independent biological replicates. The following primers were used: CXCL2, forward 5'-CAG CCTCGAGTCTCACCTGT-3'; reverse 5'-CACTCTTTG GTCCAGAGCCAT-3'. CXCL13, forward 5'-GCTCAC CTTGGA ACACCTAC-3', reverse 5'-ACACCTAGG TTGTATGCCCA-3'; CCL20, forward 5'-AAAAGATCC GTGTGCGCTGA-3', reverse 5'-CTCTGCTCCCTTATT CCCGTC-3'; CCL3, forward 5'-CGTGGAATTTGC CGTCCATAG-3', reverse 5'-GTCCTGTCCTGCTCA AGGAT-3'; IL-6, forward 5'-CCAGATAGCTGCTCA GAAGGG-3', reverse 5'-GTCTTTGCAAAAGAGAGG CCC-3'; IL1R2, forward 5'-CTATCTGGGGGACGC TGAAA-3', reverse 5'-TTCAACGCCCTACAAGGA GAC-3'; TNFSF10, forward 5'-CCGGAATGGTTGGAA GTGGTT-3', reverse 5'-GGTATGGCGGAGTTAGCACG-3'; and L32, forward 5'-GATCTTGGGCTTCACCAGAGG-3', reverse 5'-CTTGAGACACGCAGCTCCAC-3' The cycling condition used were incubation at $95^{\circ} \mathrm{C}$ for $20 \mathrm{sec}$, followed by 40 cycles of denaturation at $95^{\circ} \mathrm{C}$ for $3 \mathrm{sec}$, followed by annealing, extension and data acquisition at $60^{\circ} \mathrm{C}$ for $30 \mathrm{sec}$ using the ABI 7500 Fast Real-Time PCR system (Thermo Fisher Scientific, Inc.). The internal reference was L32 and quantification was performed using the standard curve method.

Statistical analysis. Statistical differences between groups were determined by one-way analysis of variance followed by Tukey's test for qPCR. For non-continuous histological injury score data, the Kruskal-Wallis test was used. Analysis was performed using SPSS software (version 13.0; SPSS, Inc., Chicago, IL, USA). Results are presented as the mean \pm standard error of the mean. $\mathrm{P}<0.05$ was considered to indicate a statistically significant difference.

\section{Results}

Histopathological alterations and lung injury scores in seawater aspiration-induced ALI. Histopathology images are presented in Fig. 1. The results demonstrated that seawater aspiration induced pulmonary edema, infiltration of inflammatory cells into the lung tissues and alveoli, and alveolar collapse (S group; Fig. 1C). The distilled water negative control group exhibited similar lung injury (D group; Fig. 1B). However, alterations in the $\mathrm{S}$ group were more severe compared with in the D group. Normal histopathology was observed in the lung samples of the blank control group (C group; Fig. 1A). 

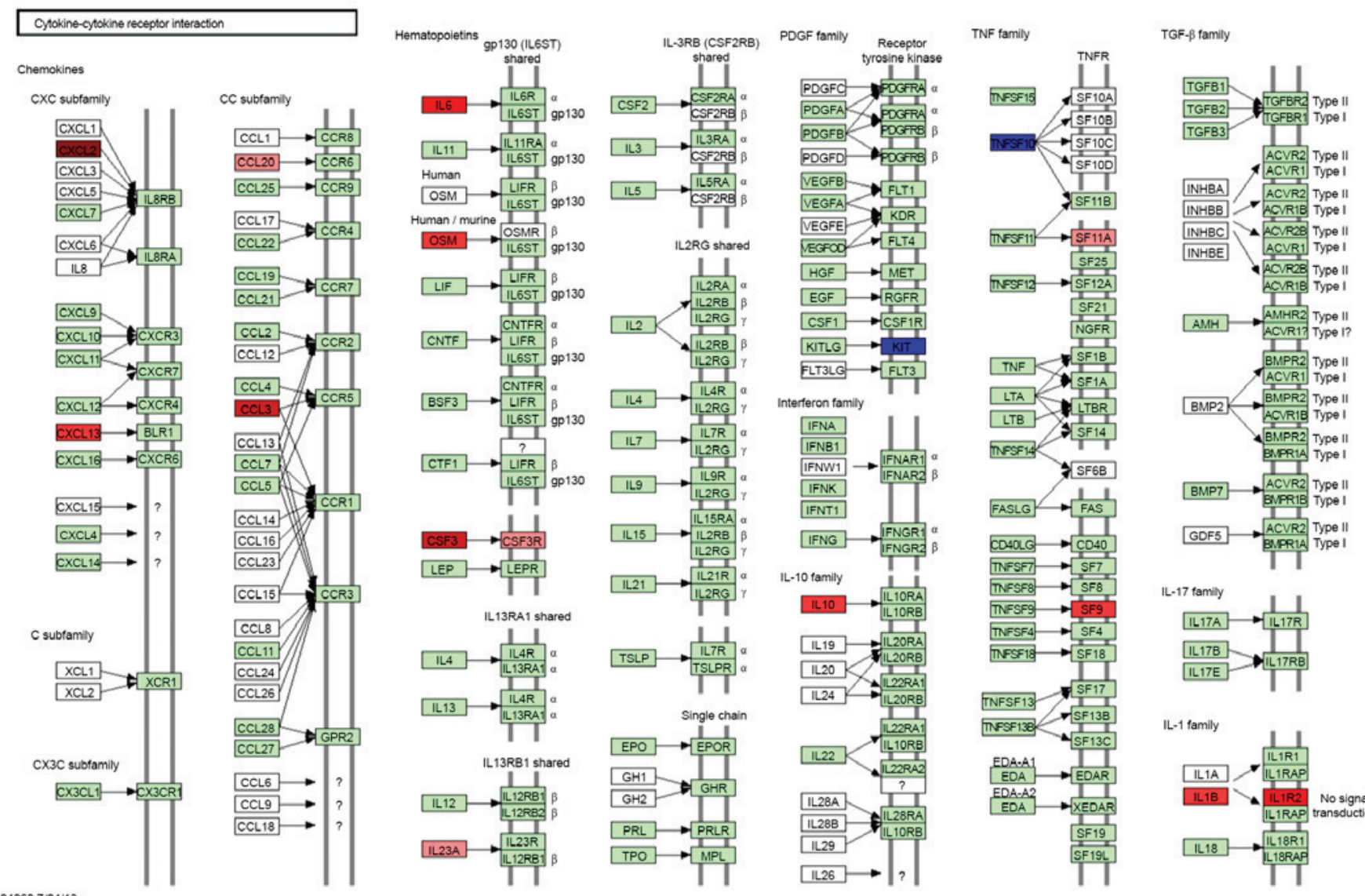

$040607 / 24 / 13$
(c) Kanehisa laboratories
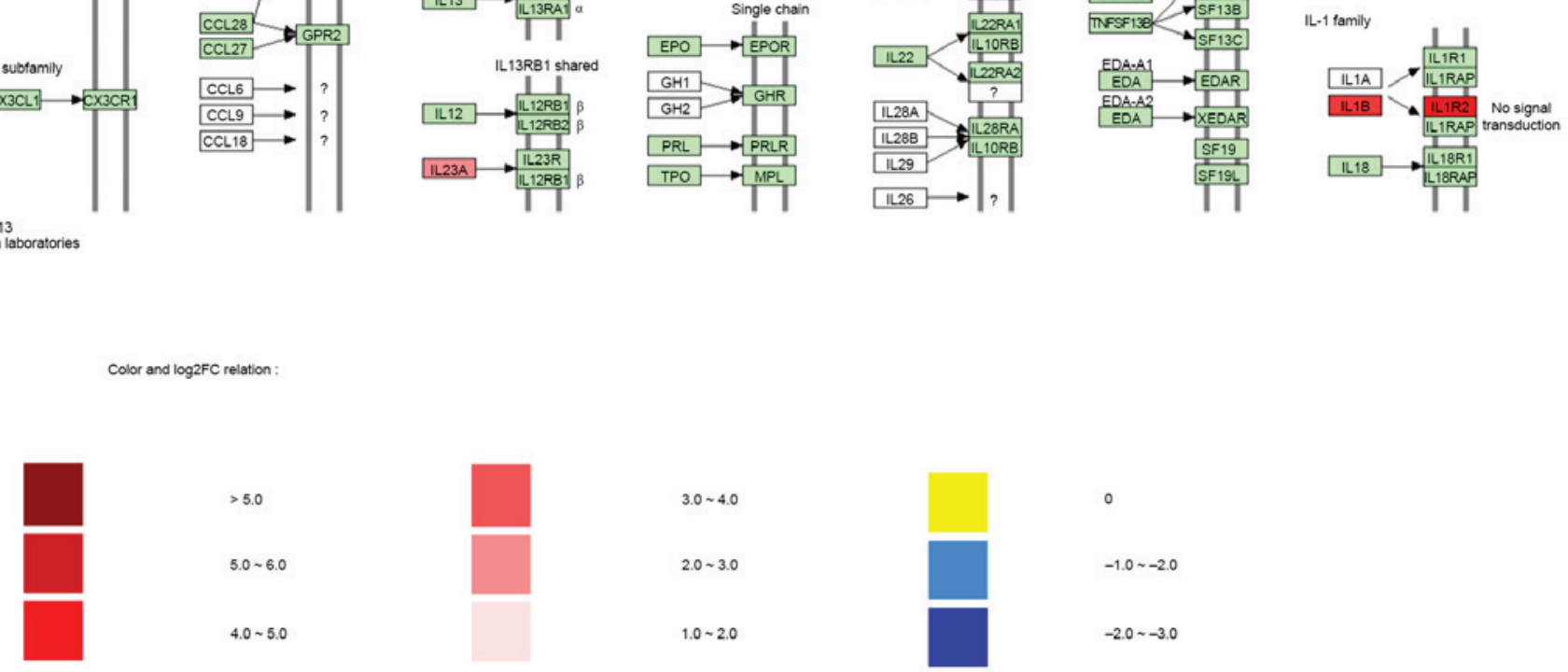

Figure 7. Genes expressed in the seawater aspiration-induced acute lung injury group related to the cytokine-cytokine receptor interaction pathway. Different colors represent different fold changes of genes.

The assigned lung injury scores of each group with regards to edema, neutrophil infiltration, hemorrhage, bronchiole epithelial desquamation and hyaline membrane formation are shown in Table I.

Lung wetldry ratios in seawater aspiration-induced ALI. To investigate the severity of pulmonary edema in the $\mathrm{S}$ group, lung wet/dry ratios were measured (Fig. 2A). The results indicated that seawater aspiration significantly increased the lung wet/dry ratios $(5.77 \pm 0.22)$ compared with the $C$ group (4.29 $\pm 0.12 ; \mathrm{P}<0.05 ; \mathrm{n}=3)$ and the $\mathrm{D}$ group $(5.2 \pm 0.15 ; \mathrm{P}<0.05$; $n=3)$.

Total protein concentrations in BALF in seawater aspiration-induced ALI. Total protein concentrations in BALF were increased in the $\mathrm{S}$ group $(793.67 \pm 30.37)$ compared with the $\mathrm{C}$ group $(228.33 \pm 28.95 ; \mathrm{P}<0.05 ; \mathrm{n}=3)$ and the $\mathrm{D}$ group (414.33 \pm 23.53 ; $\mathrm{P}<0.05$; $\mathrm{n}=3$ ) (Fig. 2B).

Gene expression analysis. A total of 29,516 genes were attained from each group by RNA-Seq short sequence read analysis, which were aligned to the Ensembl rat reference genome. A total of 277 and 250 genes in the S group were revealed to be up and downregulated respectively, as compared with the C group. In addition, there were 152 and 130 genes that were up and downregulated in the $\mathrm{S}$ group respectively, as compared with the $\mathrm{D}$ group. The top 10 up and downregulated genes are listed in Tables II and III.

Functional clusters analysis. Functional enrichment analysis of the GO and KEGG pathways was carried out with a significance threshold of 0.05 for the adjusted P-value. Identified 

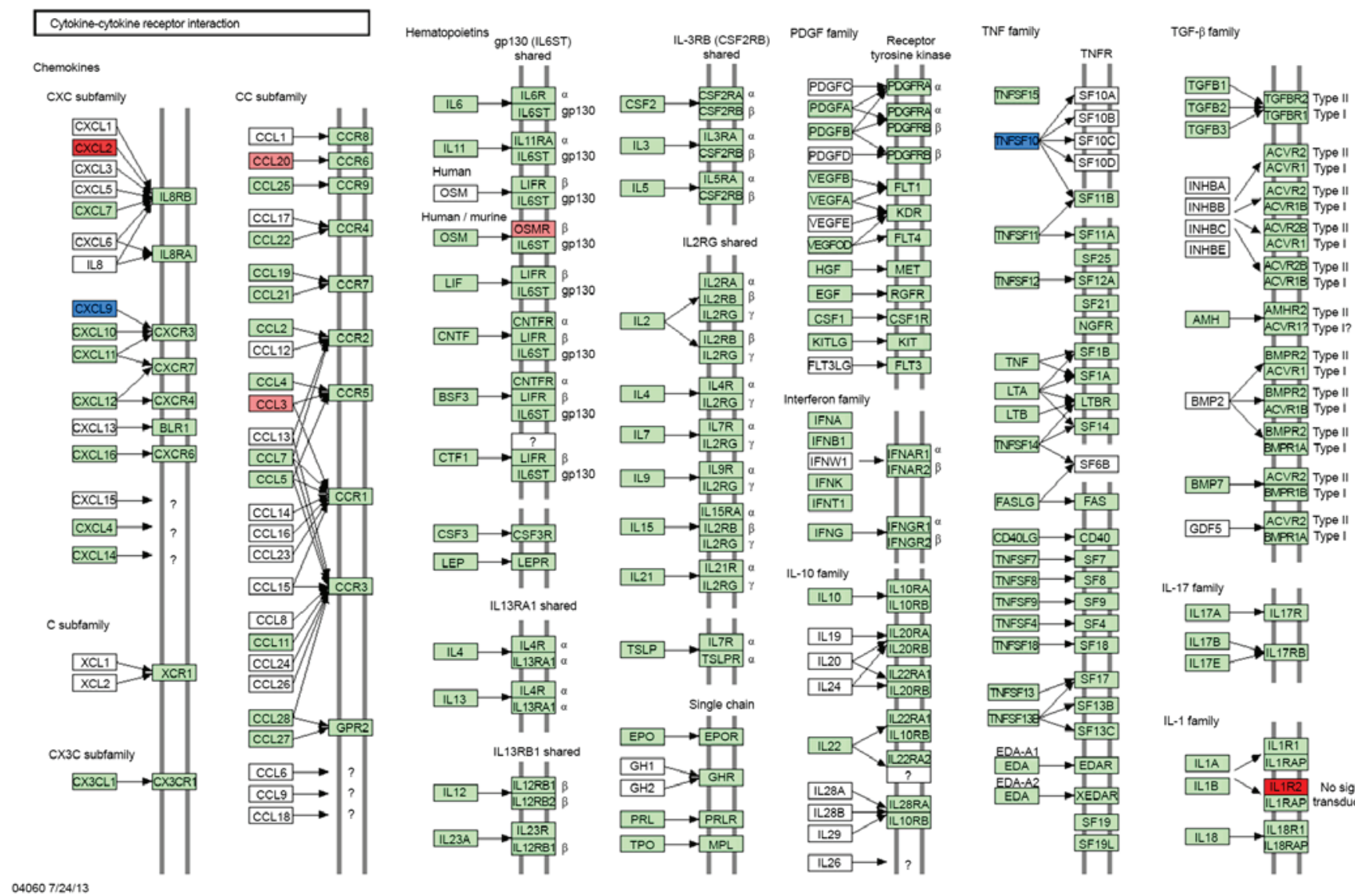

(c) Kanehisa laboratories
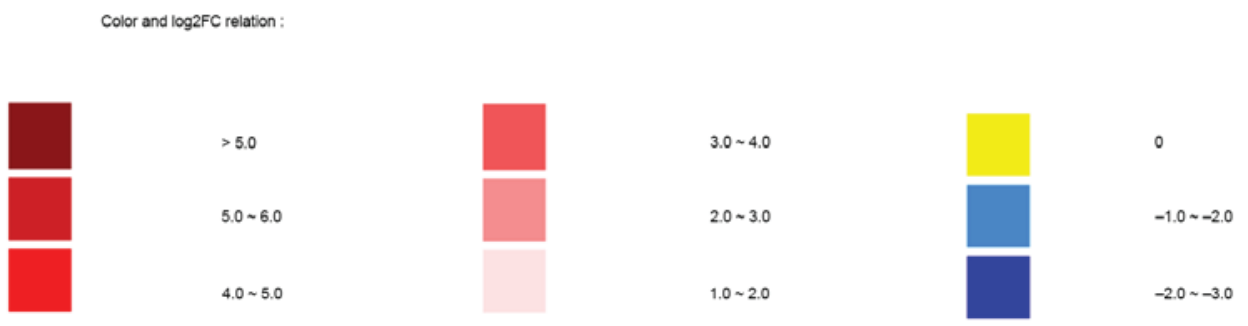

Figure 8. Genes expressed in the distilled water negative control group related to the cytokine-cytokine receptor interaction pathway. Different colors represented different fold changes of genes.

genes in the $\mathrm{S}$ group were associated with 245 specific enriched GO terms. A total of 60 of the most significant GO terms are presented in Fig. 3, of which three were associated with molecular function, four were associated with cellular component, and 53 were associated with biological process. As shown in Fig. 4, there were 80 enriched GO terms in the S and D groups. All major genes were associated with biological process, cellular component and molecular function. These genes were, for the most part, associated with neutrophil chemotaxis, immune and defense responses, and cytokine activity. Changes to these GO terms were more obvious in the $\mathrm{S}$ group compared with in the $\mathrm{D}$ group.

KEGG analysis demonstrated that several gene pathways were significantly affected in the $\mathrm{S}$ group. The top 20 selected pathways in the $\mathrm{S}$ group are presented in Fig. 5, including hematopoietic cell lineage, cytokine-cytokine receptor interaction, p53 signaling pathway, cell adhesion molecule and phagosome pathway. Selected pathways in the D group are shown in Fig. 6, including cytokine-cytokine receptor interaction, chemokine signaling pathway, toll-like receptor signaling pathway and hematopoietic cell lineage.

The cytokine-cytokine receptor interaction pathway was one of the most important pathways in the $\mathrm{S}$ and $\mathrm{D}$ groups. The cytokine-cytokine receptor interaction pathway in $\mathrm{S}$ group is shown in Fig. 7. mRNA sequencing demonstrated that certain genes were upregulated, including CXCL2, CXCL13, CCL20, CCL3, IL-6, IL-10, IL-23A, oncostatin M (OSM), colony stimulating factor (CSF), CSF3 receptor, TNF receptor superfamily member 11a (TNFSF11A) and TNFRSF9. However, other genes were downregulated, including KIT and TNFSF10. As shown in Fig. 8, the expression of certain genes associated with the cytokine-cytokine receptor interaction pathway were also altered in the D group, including CXCL2, CCL20, CCL3, OSM receptor and IL1R2, 
which were upregulated, and CXCL9 and TNFSF10, which were downregulated.

$q P C R$. qPCR is used to measure gene expression levels, and is often used to confirm the results of sequencing studies. Seven cytokine-cytokine receptor interaction pathway-related genes (CXCL2, CXCL13, CCL20, CCL3, IL6, IL1R2 and TNFSF10) were selected for qPCR. The results are shown in Table IV. The results of the gene expression profiles determined by sequencing were consistent with the results of qPCR.

\section{Discussion}

One of the serious complications of drowning is secondary acute lung injury (ALI), which is characterized by relentless deterioration and mortality $(18,19)$. Previous studies have focused on seawater aspiration-induced ALI (4-10); however, few have involved pathway and GO analyses of seawater aspiration-induced ALI. The present study is the first, to the best of our knowledge, to develop a genetic signature for seawater aspiration-induced ALI with gene expression profiles.

The histopathological analysis demonstrated that seawater aspiration-induced ALI was able to induce pulmonary edema, infiltration of inflammatory cells into the lung tissues, and alveolar collapse. These alterations were more serious in the $\mathrm{S}$ group compared with in the D group (20). To investigate the pathogenesis of seawater aspiration-induced ALI a gene expression profile analysis was conducted using KEGG and GO analyses. The results identified differential gene expression patterns among the $\mathrm{S}$ group, the $\mathrm{D}$ group and the $\mathrm{C}$ group.

GO and KEGG pathway analyses provided a global view on the function of the identified genes. The results of the GO analysis demonstrated that there were 80 enriched GO terms in the $\mathrm{S}$ and $\mathrm{D}$ groups, including biological process, cellular component and molecular function. Neutrophil chemotaxis, immune and defense responses, and cytokine activity were the most significantly enriched GO terms. These GO terms were more enriched in the $\mathrm{S}$ group compared with in the $\mathrm{D}$ group. These enriched GO terms indicated that regulation of response to external stimulus and inflammatory responses may be the main biological reactions and molecular functions in the $\mathrm{S}$ and $\mathrm{D}$ groups (21); however, these reactions were more intense in the $\mathrm{S}$ group.

The present study also revealed that hematopoietic cell lineage, cytokine-cytokine receptor interaction, p53 signaling pathway, cell adhesion molecule and phagosome pathway were significantly altered in the $\mathrm{S}$ group, whereas cytokine-cytokine receptor interaction, chemokine signaling pathway, toll-like receptor signaling pathway and hematopoietic cell lineage were significantly altered in the D group. Among these, we focused on the cytokine-cytokine receptor interaction pathway as one of the essential pathways of pathogenesis in the $\mathrm{S}$ and $\mathrm{D}$ groups. It has previously been reported that inflammation and cytokines have a key role in the pathogenesis of seawater aspiration-induced ALI $(3,21)$, as is consistent with the present study. Furthermore, the expression of genes associated with the cytokine-cytokine receptor interaction pathway were altered in the $\mathrm{S}$ group, including CXCL2, CXCL13, CCL20, CCL3, IL6, IL1R2 and TNFSF10. With regards to the inflam- matory response, lung tissue in the $\mathrm{S}$ and $\mathrm{D}$ groups expressed higher levels of chemotactic and inflammatory factors, and lower levels of members of the tumor necrosis factor ligand superfamily compared with in the $\mathrm{C}$ group. However, these gene expression changes were markedly more obvious in the $\mathrm{S}$ group compared with in the D group as detected by qPCR. In particular, several studies have reported an association between TNFSF10 and neutrophilic inflammation. It has been demonstrated that the absence of TNFSF10 [alias TNF-related apoptosis-inducing ligand (TRAIL)] results in enhancement of neutrophilic inflammation in ALI and sepsis-induced organ injury, and exogenous TRAIL can induce apoptosis of neutrophils in tissues $(22,23)$. Therefore, the downregulation of TNFSF10 may substantially contribute to the development of neutrophilic inflammation in seawater aspiration-induced ALI; however, the detailed mechanism remains unclear.

Notably, the proinflammatory cytokine IL-6 was markedly increased in the $\mathrm{S}$ group, but not in the D group compared with the $\mathrm{C}$ group. It has previously been demonstrated that IL-6 mRNA expression is increased in response to high glucose concentrations in human osteoblastic cells (24). In addition, under hyperosmotic stress, IL-6 was increased in an ex vitro human conjunctival epithelial cell model of dry-eye (25). Therefore, the differential expression of IL-6 in the S group compared with in the $\mathrm{D}$ group may be due to hyperosmotic stress.

In conclusion, the differential gene expression patterns detected in the present study suggested that activation of the cytokine-cytokine receptor interaction pathway has an important role in the pathogenesis of seawater aspiration-induced ALI. Downregulation of TNFSF10 may enhance inflammation, whereas the differential expression of IL- 6 may be due to variations in hyperosmotic stress between the $\mathrm{S}$ and $\mathrm{D}$ groups. Therefore, IL-6 may be considered a biomarker to distinguish the seawater aspiration-induced ALI group from the distilled water negative control group. However, further research is required to elucidate the mechanistic relationship and the biological implications of the overexpression or downregulation of cytokine-cytokine receptor interaction-related genes in seawater aspiration-induced ALI.

\section{Acknowledgements}

The present study was supported by the National Natural Science Foundation of China (grant no. 81270124).

\section{References}

1. van Beeck EF, Branche CM, Szpilman D, Modell JH and Bierens JJ: A new definition of drowning: Towards documentation and prevention of a global public health problem. Bull World Health Organ 83: 853-856, 2005.

2. Salomez F and Vincent JL: Drowning: A review of epidemiology, pathophysiology, treatment and prevention. Resuscitation 63: 261-268, 2004.

3. Xinmin D, Yunyou D, Chaosheng P, Huasong F, Pingkun Z, Jiguang M, Zhiqian X and Qinzhi X: Dexamethasone treatment attenuates early seawater instillation-induced acute lung injury in rabbits. Pharmacol Res 53: 372-379, 2006.

4. Ma L, Li Y, Zhao Y, Wang Q, Nan Y, Mu D, Li W, Sun R, Jin $\mathrm{F}$ and Liu X: 3,5,4'-tri-O-acetylresveratrol ameliorates seawater exposure-induced lung injury by upregulating connexin 43 expression in lung. Mediators Inflamm 2013: $182132,2013$. 
5. Zhang Y, Zhang B, Xu DQ, Li WP, Xu M, Li JH, Xie XY, Fan QX, Liu W, Mu DG, et al: Tanshinone IIA attenuates seawater aspiration-induced lung injury by inhibiting macrophage migration inhibitory factor. Biol Pharm Bull 34 1052-1057, 2011.

6. Ma L, Zhao Y, Li B, Wang Q, Liu X, Chen X, Nan Y, Liang L, Chang R, Liang L, et al: 3,5,4'-Tri-O-acetylresveratrol attenuates seawater aspiration-induced lung injury by inhibiting activation of nuclear factor-kappa B and hypoxia-inducible factor- $1 \alpha$. Respir Physiol Neurobiol 185: 608-614, 2013.

7. Liu W, Dong M, Bo L, Li C, Liu Q, Li Y, Ma L, Xie Y, Fu E, $\mathrm{Mu} \mathrm{D}$, et al: Epigallocatechin-3-gallate ameliorates seawater aspiration-induced acute lung injury via regulating inflammatory cytokines and inhibiting JAK/STAT1 pathway in rats. Mediators Inflamm 2014: 612593, 2014.

8. Li J, Xu M, Fan Q, Xie X, Zhang Y, Mu D, Zhao P, Zhang B, Cao F, Wang Y, et al: Tanshinone IIA ameliorates seawater exposure-induced lung injury by inhibiting aquaporins (AQP) 1 and AQP5 expression in lung. Respir Physiol Neurobiol 176: 39-49, 2011.

9. Han F, Luo Y, Li Y, Liu Z, Xu D, Jin F and Li Z: Seawater induces apoptosis in alveolar epithelial cells via the Fas/FasL-mediated pathway. Respir Physiol Neurobiol 182: 71-80, 2012.

10. Fan Q, Zhao P, Li J, Xie X, Xu M, Zhang Y, Mu D, Li W, Sun R, Liu W, et al: $17 \beta$-Estradiol administration attenuates seawater aspiration-induced acute lung injury in rats. Pulm Pharmacol Ther 24: 673-681, 2011

11. Hiroshima Y, Hsu K, Tedla N, Chung YM, Chow S, Herbert C and Geczy CL: S100A8 induces IL-10 and protects against acute lung injury. J Immunol 192: 2800-2811, 2014.

12. Zhang M, Dong M, Liu W, Wang L, Luo Y, Li Z and Jin F: $1 \alpha$, 25-dihydroxyvitamin D3 ameliorates seawater aspiration-induced acute lung injury via NF- $\kappa \mathrm{B}$ and RhoA/Rho kinase pathways. PLoS One 9: e104507, 2014.

13. Nosotti M, Falleni M,Palleschi A, Pellegrini C, Alessi F, Bosari S and Santambrogio L: Quantitative real-time polymerase chain reaction detection of lymph node lung cancer micrometastasis using carcinoembryonic antigen marker. Chest 128: 1539-1544, 2005 .

14. Shi Y, Zhang B, Chen XJ, Xu DQ, Wang YX, Dong HY, Ma SR, Sun RH, Hui YP and Li ZC: Osthole protects lipopolysaccharide-induced acute lung injury in mice by preventing down-regulation of angiotensin-converting enzyme 2. Eur J Pharm Sci 48: 819-824, 2013.
15. Zhou ZH, Sun B, Lin K and Zhu LW: Prevention of rabbit acute lung injury by surfactant, inhaled nitric oxide, and pressure support ventilation. Am J Respir Crit Care Med 161: 581-588, 2000.

16. Chen XJ, Zhang B, Hou SJ, Shi Y, Xu DQ, Wang YX, Liu ML, Dong HY, Sun RH, Bao ND, et al: Osthole improves acute lung injury in mice by up-regulating Nrf-2/thioredoxin 1 . Respir Physiol Neurobiol 188: 214-222, 2013.

17. Scharfman A, Hayem A, Lebas J, Laine A and Sablonnière B: Comparison of three methods for protein measurement: application to bronchoalveolar lavage fluids. Bull Eur Physiopathol Respir 16: 785-789, 1980

18. Saidel-Odes LR and Almog Y: Near-drowning in the Dead Sea: A retrospective observational analysis of 69 patients. Isr Med Assoc J 5: 856-858, 2003.

19. Gregorakos L, Markou N, Psalida V,Kanakaki M, Alexopoulou A, Sotiriou E, Damianos A and Myrianthefs P: Near-drowning: Clinical course of lung injury in adults. Lung 187: 93-97, 2009.

20. Rui M, Duan YY, Wang HL, Zhang XH and Wang Y: Differences between seawater- and freshwater-induced lung injuries. Zhongguo Wei Zhong Bing Ji Jiu Yi Xue 21: 416-420, 2009 (In Chinese)

21. Miyazato T, Ishikawa T, Michiue T and Maeda H: Molecular pathology of pulmonary surfactants and cytokines in drowning compared with other asphyxiation and fatal hypothermia. Int J Legal Med 126: 581-587, 2012.

22. McGrath EE, Marriott HM, Lawrie A, Francis SE, Sabroe I, Renshaw SA, Dockrell DH and Whyte MK: TNF-related apoptosis-inducing ligand (TRAIL) regulates inflammatory neutrophil apoptosis and enhances resolution of inflammation. J Leukoc Biol 90: 855-865, 2011.

23. Beyer K, Poetschke C, Partecke LI, von Bernstorff W, Maier S, Broeker BM and Heidecke CD: TRAIL induces neutrophil apoptosis and dampens sepsis-induced organ injury in murine colon ascendens stent peritonitis. PLoS One 9: e97451, 2014.

24. Garcia-Hernández A, Arzate H, Gil-Chavarria I, Rojo R and Moreno-Fierros L: High glucose concentrations alter the biomineralization process in human osteoblastic cells. Bone 50: 276-288, 2012.

25. Kim JH, Kang SS, Kim ES, Kim JY, Kim MJ and Tchah H: Osmoprotective effects of supplemental epidermal growth factor in an ex vivo multilayered human conjunctival model under hyperosmotic stress. Graefes Arch Clin Exp Ophthalmol 251: 1945-1953, 2013. 